\title{
Droplet-on-cantilever approach for determining the mass of magnetic particles
}

\author{
Wilson Ombati Nyang'au ${ }^{1,2}$, Andi Setiono ${ }^{1,3}$, Prabowo Puranto ${ }^{1,3,4}$, Maik Bertke ${ }^{1}$, Hutomo Suryo Wasisto ${ }^{1}$, \\ Thilo Viereck ${ }^{5}$, Harald Bosse ${ }^{4}$, Erwin Peiner ${ }^{1}$ \\ 1 Institute of Semiconductor Technology (IHT) and Laboratory for Emerging Nanometrology \\ (LENA), Technische Universität Braunschweig, Braunschweig, Germany \\ ${ }^{2}$ Department of Metrology, Kenya Bureau of Standards, Nairobi, Kenya \\ ${ }^{3}$ Research Center for Physics, Indonesian Institute of Sciences (LIPI), Banten, Indonesia \\ ${ }^{4}$ Precision Engineering Division, Physikalisch-Technische Bundesanstalt (PTB), Braunschweig, \\ Germany \\ ${ }^{5}$ Institut für Elektrische Messtechnik und Grundlagen der Elektrotechnik (EMG), Technische \\ Universität Braunschweig, Braunschweig, Germany
}

E-mail:wilombat@tu-braunschweig.de, a.setiono@tu-braunschweig.de,m.bertke@tubraunschweig.de, h.wasisto@tu-braunschweig.de, t.viereck@tu-braunschweig.de, harald.bosse@ptb.de,e.peiner@tu-braunschweig.de

\begin{abstract}
In this study, we present a technique that involves depositing a droplet laden with microparticles $(\mu \mathrm{Ps})$ onto a defined sensing area on a microcantilever sensor. This process entailed the optimization of dispensing parameters (pressure $p$ and pulse time $t$ ) to the generation of a small water droplet volume $\left(V_{d}\right.$ $=53.0 \pm 2.72 \mathrm{pL}$ ). Subsequently, the evaporation trends of the particle carrier fluid, i.e., the water droplets on the sensor were monitored and a total evaporation time of $t_{\mathrm{ev}}=40 \pm 2 \mathrm{~s}$ was determined. By using monodispersed particles in water, i.e., magnetic polystyrene matrix particles (MPS) and poly methyl methacrylate (PMMA) $\mu \mathrm{Ps}$, and adsorbing them on a dynamic cantilever sensor, the mass and number of these $\mu \mathrm{Ps}$ were measured and determined comparatively using both resonant frequency measurements and scanning electron microscopy (SEM), respectively. As a result, we have observed and reported monolayer particles assembled on the sensor with a particles count of at least 203.
\end{abstract}

Key words: Microcantilever, droplet, mass, magnetic particles, resonant frequency

\section{Introduction}

Over the years, there has been a growing dynamic demand in determining the mass of liquidborne particles. Much of such interests and applications have increasingly been exploited in fields such as medicine and biological sciences, optics, printing and coating, and electronics manufacture. In medicine, for instance, magnetic particles have been used for cell sorting and separation, and as contrast reagents in magnetic resonance imaging (MRI). Moreover, droplet dispensing has been utilized in drug discovery and delivery as well as hyperthermia treatment [13]. The mass of such particles plays a critical role in these applications. However, most available equipment for determining their masses, e.g. mass spectrometers and microchannel resonators [4], utilize complicated technology and are quite expensive. Thus, a low-cost and direct approach involving dipping of a cantilever [5] into a magnetic particles solution to adsorb such particles has also recently been employed. This approach, however, yields a low quality factor $(Q \sim 180)$ [5] due to randomized adsorption of the particles on the whole cantilever surface. Contrarily, our previous study has utilized a liquid-dispensing mechanism to sub-sample and load water droplets $(\sim 3.44 \mu \mathrm{L})$ laden with and without particles, i.e., $\sim 1.96 \mu \mathrm{m}$ poly methyl methacrylate (PMMA) and $\sim 4.97 \mu \mathrm{m}$ polystyrene (PS) from Sigma-Aldrich Inc., onto a silicon-based cantilever sensor (see Figure 1) to achieve a $Q \sim 1224$ [6]. As a selfvalidating process, particle-laden droplets were also deposited on surface-treated silicon substrates and upon solvent evaporation, particle count estimations were performed using scanning electron microscopy (SEM). The particle counting process was, however, curtailed due to the observed clustering of non- 
uniform multilayered particles at the edges of the droplet after solvent evaporation [6].

The current study has therefore been intended to optimize dispensing parameters so as to generate and deposit a small droplet $(<3.44$ $\mu \mathrm{L})$ on a microcantilever using a liquid dispenser (1500XL, Nordson EFD Inc., USA); to monitor and determine the total evaporation time $\left(t_{\mathrm{ev}}\right)$ and trends thereof for water droplets of known volume $\left(V_{d}\right)$; and also to determine the mass or number of monodispersed microparticles ( $\mu \mathrm{Ps})$. The $\mu \mathrm{Ps}$ used were mainly magnetic polystyrene matrix microparticles (MPS, Micromod $\mathrm{GmbH}$ ), and PMMA microparticles (Sigma-Aldrich Inc.). The two test $\mu \mathrm{Ps}$ have comparable diameters ( $\sim 2 \mu \mathrm{m})$ and densities; but, have contrasting magnetic properties, i.e., MPS are magnetic whereas PMMA particles are non-magnetic. Magnetic polymer particles (e.g., MPS) are synthesized by embedding magnetic nanoparticles (pure metal such as Fe, Co and $\mathrm{Ni}$ or their oxides) into a non-magnetic spherical (polymer) matrix [7]. The $\sim 2 \mu \mathrm{m}$ MPS, for instance, have a polymer core (PS) and magnetic shell consisting of iron oxide nanoparticles. Hence, MPS exhibit a superparamagnetic behavior. However, similar to silicon, PMMA has magnetic susceptibility of less than 1 and is therefore diamagnetic in nature. The essence of using these two $\mu \mathrm{Ps}$ was to compare the results and assess the performance of our measurement approach.

\section{Substrate preparation and sensor fabrication}

Initially, silicon substrates were diced from $n$ type (100) silicon wafers (Siegert Wafer $\mathrm{GmbH}$, Germany) with resistivity and thickness of $1-10 \Omega \mathrm{cm}$ and $275 \pm 15 \mu \mathrm{m}$, respectively, into $\sim 15 \times 15 \mathrm{~mm}^{2}$ and $\sim 30 \times 30 \mathrm{~mm}^{2}$ pieces. The latter were used for fabrication of cantilever sensors whereas the former, upon surface treatment with oxygen plasma for $30 \mathrm{~s}$, were used as particle adsorbing medium. All the diced samples were cleaned by (a) boiling them for $5 \mathrm{~min}$ in an oxidant solution containing a mixture of sulfuric acid $\left(\mathrm{H}_{2} \mathrm{SO}_{4}\right.$, $96 \%)$ and hydrogen peroxide $\left(\mathrm{H}_{2} \mathrm{O}_{2}, 30 \%\right)$ in a volume ratio of $1: 1$, (b) immersing in a water bath for $5 \mathrm{~min}$, (c) thoroughly rinsing with deionized water and (d) blow-drying with nitrogen [6] prior to surface treatment or fabrication processes. All the cantilever sensors were fabricated utilizing $n$-type (100) silicon (as base material) and bulkmicromachining technology, discussed elsewhere [8, 9]. The fabrication process mainly comprises thermal oxidation, photolithography, dopant diffusions (boron and phosphorus), etching processes (i.e., HF dip and cryogenic etching), contact holes formation, and metallization. Upon fabrication and prior to use, the cantilever sensors were thoroughly cleaned by immersing or soaking them in acetone, rinsing with deionized water and freely drying them at ambient conditions. Thereafter, we characterized the fabricated cantilever(s) by determining for instance their resonant frequency $f_{0}$ and geometry (e.g. thickness $h_{\mathrm{c}}$, length $L$ and width $w$ ).

\section{Experimental design and procedure}

\subsection{Optimization of dispensing parameters and measurement of droplet volume}

Large droplets on microstructures are bound to spill-off and sometimes move towards the clamped-end of the cantilever [6]. Furthermore, since the target droplet deposit area on the cantilever is limited and small, it is therefore necessary to optimize the dispensing parameters such as output air pressure $(p)$ and pulse duration $(t)$ and dispensing tip size so as to generate small droplets. The knowledge of the droplet size is thus helpful in precisely depositing and localizing the droplet on a defined region of the cantilever. In our case, the target droplet deposit point on the cantilever was $\sim 400 \mu \mathrm{m}$ from the free-end. For optimizing $p$ and $t$, and determining the droplet volume $V_{d}$ (Figure 3 ), deionized water was used as the test liquid. Due to the fragility of the cantilever sensors, we initially dispensed single water droplets on treated bulk $\mathrm{Si}$ substrates so as to estimate optically the droplet volume $\left(V_{d}=2.78 \pm 0.28 \mathrm{~nL}\right)$. This was done by capturing droplet images, and analyzing them using the low-bond axisymmetric drop shape (LB-ADSA) method [10]. For comparability and better precision, $V_{d}$ was also determined from the cantilever-based resonant frequency shift $\Delta f$ measurements.

The water droplet was deposited on the cantilever using a liquid dispenser fitted with: a barrel (filled with deionized water), a dispensing needle (with internal diameter $\approx$ $0.10 \mathrm{~mm}$ ) and air pressure supply. The desired output air pressure and pulse time were set and adjusted accordingly, and initially the test liquid was freely dispensed on a test paper 
instead of a test sample. At this stage, the dispensing tip would be aligned perpendicularly on top of the test surface (see Figure 1). The positioning and alignment of the tip on the sample surface were accomplished using the 3D coordinate micropositioning system. To minimize the errors due to misalignment and possibility of breaking the fragile cantilever, a USB digital microscope (Mz-902, Oowl Tech Ltd.) was further integrated in the alignment process in order to provide a clear view.

Before and after depositing the water droplet on the cantilever, the resonant frequency $f_{0}$ was measured (fundamental in-plane vibration mode). The actuation and vibration sensing mechanisms of the cantilever consisted of a piezoelectric shaker and a U-shaped piezoresistive Wheatstone bridge (WB), respectively. A Lock-in amplifier (MFLI, Zurich Instruments Ltd.) was used to supply the WB voltage $\left(1 \mathrm{~V}_{\mathrm{dc}}\right)$ and the excitation signal $\left(9.9 \mathrm{~V}_{\mathrm{pp}}\right)$. The MFLI was connected and controlled (by software) from a computer, and connections to and from our electronics were done through coaxial cables and SMA connectors.

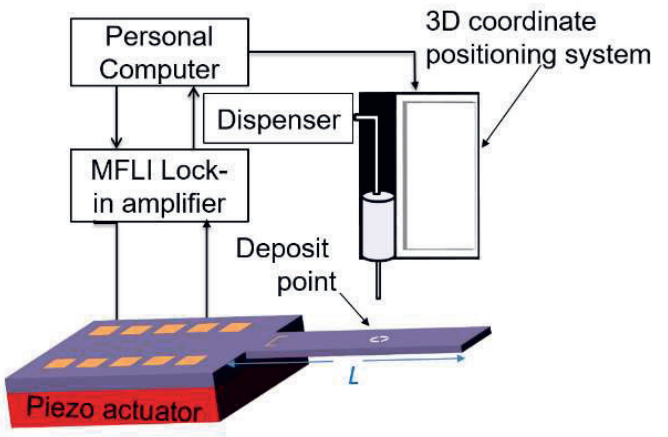

Figure 1: (a) Droplet deposition system and resonant frequency measurement set-up and (b) dispensed droplet on a cantilever.

During droplet dispensing, the tip was moved in close proximity of the cantilever sensing area and an output pressure $p$ was applied for a specified pulse duration $t$. Consequently, the droplet mass $m_{\mathrm{d}}$ would be determined at a point $x$ from the fixed-end of the cantilever of length $L$. With the measured resonant frequency shift $\Delta f$, the effective mass of the cantilever $m_{\text {eff }}$ and the normalized mode-shape function $\phi\left(x_{\mathrm{md}}\right)$, the drop mass can be calculated using Equations (1a) and (1b) [11]. The parameter $\lambda$ (1b) represents a modal constant, which is about 1.875 for the first mode.

By varying dispensing time and output pressure, various droplet sizes were obtained. It was clearly observed (Figure 2) that for the same dispensing pressure (60 mbar), the size of the water droplet increased with the pulse duration $(t)$. By multiplying $m_{\mathrm{d}}$ with the water density $\left(\rho_{\mathrm{w}}=1 \mathrm{gcm}^{-3}\right)$, we obtain the drop volume. Obviously, a small droplet size $V_{d}=$ $53.0 \pm 2.7 \mathrm{pL}$ was measured for $t=10 \mathrm{~ms}$ with $p=60$ mbar. Similarly, $V_{\mathrm{d}}=76.8 \pm 7.7 \mathrm{pL}$ corresponds to $t=50 \mathrm{~ms}$ for $p=60 \mathrm{mbar}$. Consequently, we used low output air pressure (60 mbar) and the shortest pulse duration $(10 \mathrm{~ms})$ to generate and realize all the subsequent droplet volumes. The quality factor before $\left(Q_{b} \approx 2100 \pm 210\right)$ and after $\left(Q_{a}=\right.$ $181 \pm 43$ ) depositing the water droplet shows a significant reduction due to damping of the resonator. By assuming a spherical-shaped droplet of $V_{\mathrm{d}} \approx 53 \mathrm{pL}$, a diameter $\sim 46.6 \mu \mathrm{m}$ was computed; which is much smaller than the maximum width of the cantilever sensor $(w \approx$ $170 \mu \mathrm{m})$.

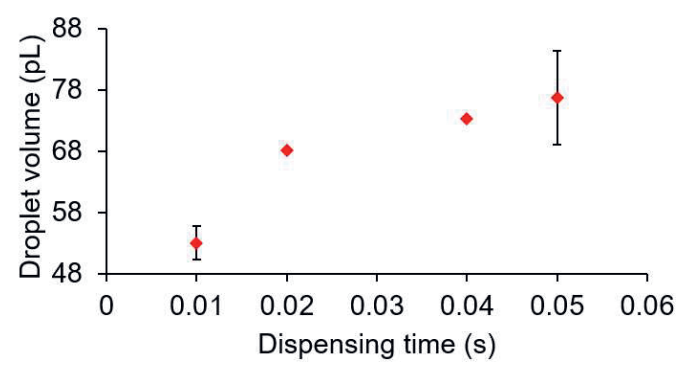

Figure 2: Volume of water droplets dispensed with a needle tip (diameter $0.10 \mathrm{~mm}$ ) on a cantilever under constant air pressure (60 mbar) at ambient conditions $(T \approx 22.2$ $\pm 0.5^{\circ} \mathrm{C} ; \mathrm{rH} \approx 30 \pm 3 \%$ )

$$
\begin{gathered}
m_{\mathrm{d}}=2 m_{\mathrm{eff}} \frac{\Delta f}{f_{0}} \phi^{2}\left(x_{\mathrm{m}_{\mathrm{d}}}\right) \\
\phi\left(x_{\mathrm{m}_{\mathrm{d}}}\right)=\frac{1}{2}\left[\cos \left(\lambda \frac{x}{L}\right)-\cosh \left(\lambda \frac{x}{L}\right)-\frac{\cos (\lambda)+\cosh (\lambda)}{\sin (\lambda)+\sinh (\lambda)}\left(\sin \left(\lambda \frac{x}{L}\right)-\sin \left(\lambda \frac{x}{L}\right)\right)\right]
\end{gathered}
$$




\section{Evaporation dynamics of small droplets on microcantilever surface}

Water droplets on a cantilever sensor are bound to evaporate at ambient conditions. In our study, the loss of water droplet mass from the sensing surface was monitored by measuring the shift in resonant frequency over time. This was necessary to determine the droplet evaporation time $t_{\mathrm{ev}}$, and the minimum particle adsorption or waiting time prior to resonant frequency measurement. Therefore, in determining $t_{\mathrm{ev}}$, the loss of droplet mass was initially monitored over time under ambient conditions through an offline resonant frequency measurement system (using a MFLI lock-in-amplifier). It should be noted (Figure 3) that as the water droplet was deposited on the cantilever sensor, an immediate shift in resonant frequency was observed, i.e., from $f_{0}$ $\sim 205.113 \mathrm{kHz}$ to $f_{\mathrm{r}} \sim 203.843 \mathrm{kHz}$ ), which corresponds to the added mass by the droplet. The total time taken for the cantilever to restore from $f_{\mathrm{r}}$ to $f_{0}$ is equivalent to the drop evaporation time, $t_{\mathrm{ev}}=40 \pm 2 \mathrm{~s}$.

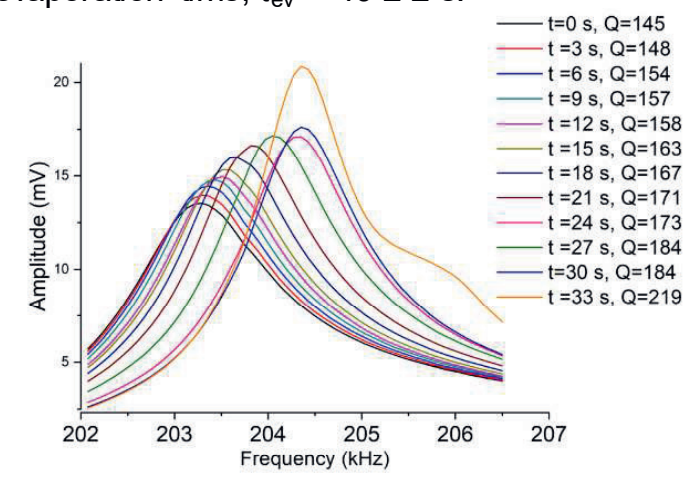

(a)

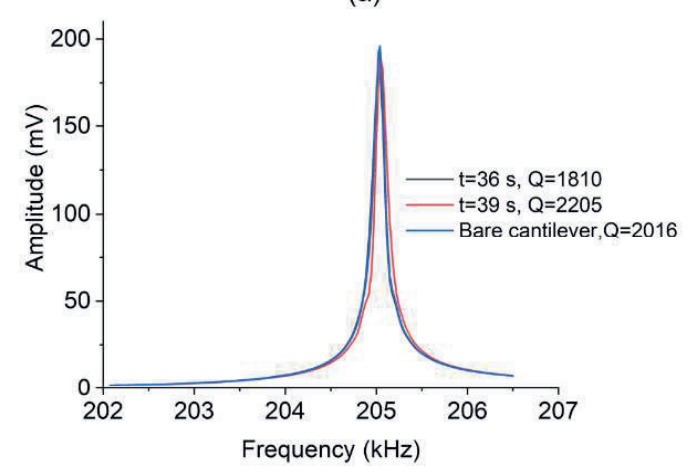

(b)

Figure 3: Offline monitoring and measurement of frequency shifts with time for an evaporating water droplet on a cantilever sensor at ambient conditions.
It has clearly been observed (Figure 3) that once the water droplet is loaded onto the cantilever, the frequency $\left(f_{0}\right)$ shifts for $\sim 5 \mathrm{~s}$ to a minimum whereupon the sensor momentarily stabilizes after droplet-deposit impact before uniformly and exponentially losing the water into the ambient atmosphere. Afterwards, it was however observed that the remaining water on the sensor surface evaporates rapidly and instantaneously (within $\sim 3 \mathrm{~s}$ ); which then culminates to a rapid shift of frequency back to $f_{0}$. This evidently shows an instantaneous change (increase) in the evaporation rate. Contrarily, Arcamone et al. [12] observed a decrease in the evaporation rate with time during the evaporation process.

A spherical droplet cap is characterized by four different parameters: the drop height $\left(h_{\mathrm{d}}\right)$, the contact radius $\left(r_{\mathrm{c}}\right)$, the radius of the sphere forming the spherical cap $\left(R_{\mathrm{S}}\right)$, and the contact angle ( $\theta$, in radians). By geometry, the relationships between the two radii $\left(r_{\mathrm{c}}\right.$ and $\left.R_{\mathrm{s}}\right)$, $\theta$, and the volume of the spherical cap $\left(V_{c}\right)$ at any instant in time are [13]:

$$
\begin{aligned}
& r_{\mathrm{c}}=R_{\mathrm{s}} \sin \theta \\
& V_{\mathrm{c}}=\pi R_{\mathrm{s}}{ }^{3} \frac{\beta}{3 \sin ^{3} \theta}
\end{aligned}
$$

where $\beta=2-3 \cos (\theta)+\cos ^{3}(\theta)$. The height of the spherical cap above the solid surface is given by: $h_{\mathrm{d}}=R_{\mathrm{s}}[1-\cos (\theta)]$ or $h_{\mathrm{d}}=r_{\mathrm{c}} \tan (\theta / 2)$. During droplet evaporation, the volume of the droplet on the solid surface at any time $t$ can be computed using the following equation [13]:

$$
\left.V_{c}^{2 / 3}=V_{c, i}^{2 / 3}-\frac{2}{3} K f(\theta) \cdot\right) t
$$

where, $V_{c, i}$ denotes the initial droplet volume (at the time $t=0 \mathrm{~s}$ ) and $2 / 3 K f(\theta) t$ represents the volume decrease with time. The function $f(\theta)=-\cos \{\theta /[2 \ln (1-\cos \theta)]\}$ is for the contact angle and for constant contact-angle evaporation mode: $f(\theta)=f\left(\theta_{0}\right)$, where $\theta_{0}$ is the contact angle prior to evaporation; and $K \approx 12.37467091 D \Delta c /\left(\rho \beta^{1 / 3}\right)$, where the symbols $D, \Delta c$ and $\rho$ denote the diffusivity of the vapor molecules in the gas (air) $\left(\mathrm{cm}^{2} \mathrm{~s}^{-1}\right)$, the difference between the saturation concentration of water vapor at the sphere surface $c_{\mathrm{s}}\left(\mathrm{gcm}^{-3}\right)$ and the ambient vapor concentration at infinite distance $c_{\infty}\left(\mathrm{gcm}^{-3}\right)$ to the droplet, and the liquid density $\left(\mathrm{gcm}^{-3}\right)$, respectively. For a water droplet, $\rho_{1}=\rho_{\mathrm{w}} \approx$ $1 \mathrm{gcm}^{-3}, D \approx 0.282 \mathrm{~cm}^{2} \mathrm{~s}^{-1}$ and $c_{\mathrm{S}}=p_{\mathrm{v}} \times M_{\mathrm{w}} /(R T)$ [13] is $\sim 1.7081 \times 10^{-4} \mathrm{gcm}^{-3}$ given a molecular mass $M_{w}$ of $18.01528 \mathrm{gmol}^{-1}$, the gas constant 
$R=8.314 \mathrm{~J}(\mathrm{~mol} \cdot \mathrm{K})^{-1}$ and the vapor pressure $p_{v}$ in air of $22.2 \mathrm{mmHg}(\cong 29.6 \mathrm{mbar})$ at a temperature $T \approx 24^{\circ} \mathrm{C}$ [14]. Based on Equation (4), we can therefore estimate the total time taken for the liquid droplet to completely vanish from the surface of a cantilever sensor $\left(\theta \sim 40^{\circ}\right)$ to be about $38.8 \mathrm{~s}$, which agrees very well with experimental droplet evaporation time $\left(t_{\mathrm{ev}} \approx 40 \mathrm{~s}\right)$. The quality factor was also observed to rapidly increase from about 200 (see Figure 3a) to more than 1800 (Figure 3b) towards the last phase of evaporation.

$$
t_{\mathrm{ev}}=\frac{3 / 2 v_{\mathrm{c}, \mathrm{i}}{ }^{2 / 3}}{K f(\theta)}
$$

\section{Particles adsorption and measurements}

\subsection{Adsorption of particles}

A particle-laden droplet ( $\sim 53 \mathrm{pL})$ was sampled and dispensed on Si (100) substrates, and cantilever sensors (at a point $\sim 400 \mu \mathrm{m}$ from the free end). In this study, the approximate particle concentrations used were $\sim 1 \%$ (w/v) and $\sim 1.25 \%(w / v)$. Before drawing a test particle solution, the aqueous $\mu \mathrm{Ps}$ suspensions were thoroughly mixed and agitated in an ultrasonic bath to homogenize the particle solution. Consequently, we assumed a uniform mass concentration in the sampled droplet(s). Upon dispensing the droplet containing $\mu \mathrm{Ps}$, a time lapse equivalent to $t_{\mathrm{ev}}$ was allowed for the water to completely evaporate and particles to adsorb on the sensing surface (Figure 4).

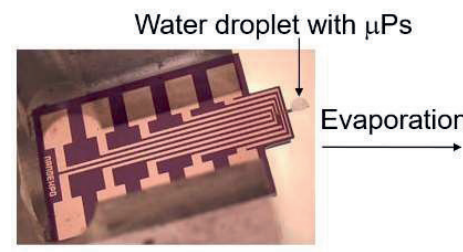

(a)

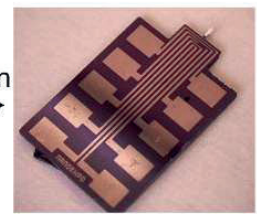

(b)
Figure 4: (a) Particle-laden droplet on a cantilever. Solvent evaporation leads to (b) particle adsorption on sensor.

\subsection{Resonant frequency measurements}

The wet mass and dry mass of the droplet can be measured immediately after depositing the droplet and evaporating the droplet, respectively. In both cases, a shift in resonant frequency is observed. Firstly, in case of a wet drop, the resonant frequency of the unloaded cantilever $\left(f_{0}\right)$ shifts to $f_{\mathrm{r} 1}$ as a droplet lands on a sensor (i.e., cantilever + water $+\mu \mathrm{Ps}$ ). Secondly, after a time lapse of $t_{\mathrm{ev}}$ the water is expected to completely vanish or evaporate from the sensor thereby shifting the resonant frequency $\left(f_{\mathrm{r} 1}\right)$ to $f_{\mathrm{r} 2}$ (i.e., resonant frequency of cantilever - water $+\mu \mathrm{Ps})$. The mass $m_{\mathrm{p}}$ of the adsorbed $\mu \mathrm{Ps}$ can therefore most conveniently be computed (Equation 1) from the frequency shift of $f_{\mathrm{r} 1}$ to $f_{\mathrm{r} 2}$, i.e., $\Delta f=f_{\mathrm{r} 2}-f_{\mathrm{r} 1}$. Similarly, the mass of the particle carrier fluid (i.e., water) can at the same time be determined from the frequency changes of $f_{\mathrm{r} 1}$ (wet mass) to $f_{\mathrm{r} 2}$ (dry mass); which nearly corresponds to the previously discussed evaporation trends. In this study, however, since evaporation time and droplet size have previously been determined, only $f_{\mathrm{r} 2}$ and $f_{0}$ were therefore of essence. Subsequently, the mass of the adsorbate (Figure 5a) would then be determined from the frequency shift (Figure $5 b$ ) using Equation (1). For a mass at point $x \approx$ $600 \mu \mathrm{m}$ and $x \approx L=1000 \mu \mathrm{m}$ (see Figure 1), the value of $\phi\left(x_{\mathrm{md}}\right)$ is about -0.46 and -1 , respectively.
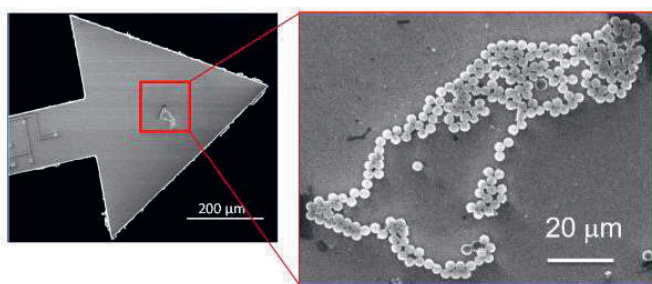

(a)

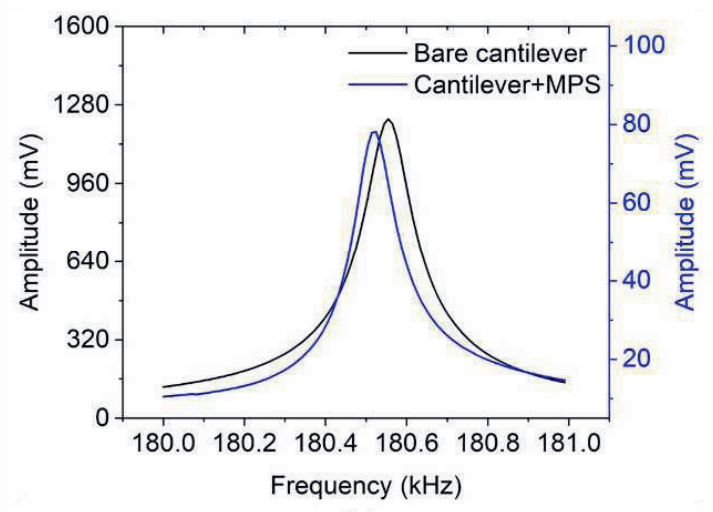

(b)

Figure 5: (a) SEM image of a triangular cantilever with MPS $\mu P s$, and a completely self-assembled monolayer of $\mu P s$ (inset). (b) Resonant frequency measurement of a bare cantilever with and without MPS $\mu$ Ps.

Furthermore, given the knowledge of the particle sizes, i.e. $1.83 \pm 0.03 \mu \mathrm{m}$ (MPS) and $1.90 \pm 0.05 \mu \mathrm{m}$ (PMMA), together with their respective densities of $1.1 \mathrm{gcm}^{-3}$ (MPS) and 
$1.18 \mathrm{gcm}^{-3}$ (PMMA), the corresponding number of adsorbed particles can be estimated from the mass ratio of the adsorbate to the respective single particle. The mass of the latter is calculated from the product of particle volume (assuming spherical-shape) and particle density.

\subsection{Microscopic particle analysis}

Particles dispersed in a water droplet can assemble themselves on a solid surface as the solvent evaporates. Depending on solidsurface properties and particle concentration, particles may form monolayers or multilayers or a monolayer segment (at the center) combined with a cluster-ring of multilayers [6]. In the present study, $\mu \mathrm{Ps}$ were deposited and adsorbed on a hydrophilic Si surface. The latter is highly adhesive and allows convective flow of fluid carrier thereby leading to the selfassembly of particles upon solvent evaporation. Initially, a particle count estimate from SEM micrographs for single droplets deposited on Si substrates was found to be $104470 \pm 420$. However, by tuning the particle concentration and droplet size, a small particle assembly was realized on the cantilever sensors.

Firstly, the particles were adsorbed on the cantilever and the resonant frequency thereof was measured (cf. Figure 5b). Secondly, the same sample was mounted on the SEM and imaging of particles was done. Subsequently, a particles count would be done manually or with ImageJ [15]. Lastly, prior to the next measurement series, the cantilever would be cleaned as earlier prescribed. Typically, a monolayer of particles was adsorbed on the sensor (Figure 6a) with a particles count of 203 and in some instances, a monolayer plus cluster(s) of non-uniform multilayers would be observed (Figure 6b). In an instance like the latter, only particles on the top layer can be counted with certainty to give minimum number of the adsorbate (e.g. > $300 \mu \mathrm{Ps}$, see Table 1). In Table 1, we present a summary of particle counts (from SEM imaging) and compare the same with the number of particles calculated from resonant frequency measurements. For the same adsorbate, the number of particles due to both point mass $\left(\Phi\left(x_{\mathrm{md}}\right)>-1\right)$ and distributed mass $\left(\Phi\left(x_{\mathrm{md}}\right) \approx-1\right)$ was calculated based on Equation (1). From both mass distributions, disparities in the number of particles were observed. Nonetheless, the number of $\mu \mathrm{Ps}$ due to distributed mass was

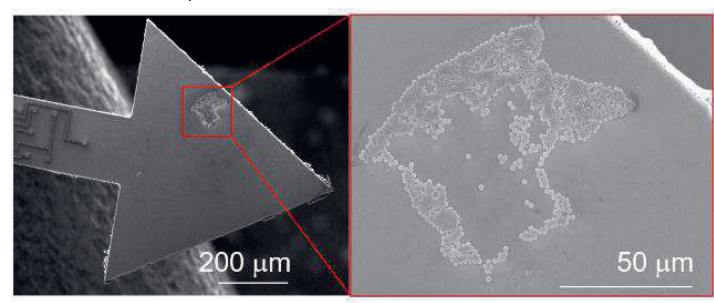

(a)

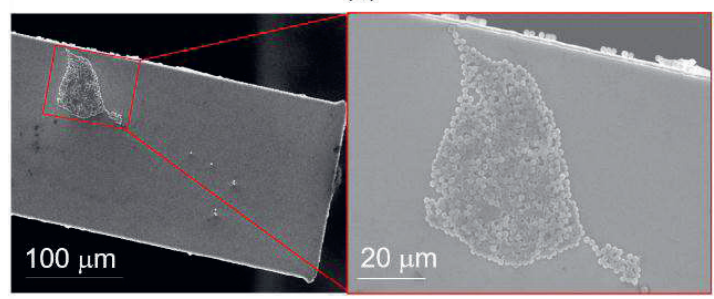

(b)

Figure 6: SEM images of adsorbed $\mu P s$ on cantilevers with triangular (a) and rectangular (b) free-ends. Particle distribution profiles consisting of monolayer and mono/multilayers are in inset, respectively, in (a) and (b).

Table 1: A comparison of number of $\mu$ Ps from resonant frequency shifts and SEM imaging

\begin{tabular}{|c|c|c|c|c|c|c|}
\hline \multirow{3}{*}{$\begin{array}{l}\mu \mathrm{Ps} \text { type/ } \\
\text { concentration } \\
(\% \mathrm{w} / \mathrm{V})\end{array}$} & \multirow{3}{*}{$\begin{array}{l}\text { Cantilever sensor free- } \\
\text { end configuration and } \\
\text { target point of } \mu \mathrm{Ps} \\
\text { deposition }\end{array}$} & \multicolumn{4}{|c|}{ Resonant frequency measurements } & \multirow{3}{*}{$\begin{array}{l}\text { SEM } \\
\mu P S \\
\text { count }\end{array}$} \\
\hline & & \multirow[t]{2}{*}{$\begin{array}{l}f_{0} \\
{[\mathrm{~Hz}]}\end{array}$} & \multirow[t]{2}{*}{$\begin{array}{l}\Delta f \\
{[\mathrm{~Hz}]}\end{array}$} & \multicolumn{2}{|c|}{$\begin{array}{c}\text { Calculated number of } \\
\mu \mathrm{Ps}\end{array}$} & \\
\hline & & & & $\begin{array}{l}\text { with point } \\
\text { mass }\end{array}$ & $\begin{array}{c}\text { with } \\
\text { distributed } \\
\text { mass }\end{array}$ & \\
\hline \multirow{3}{*}{ MPS ( 1) } & Triangular $(x \sim 600 \mu \mathrm{m})$ & 181452 & -15.2 & 1375 & 292 & 203 \\
\hline & Triangular $(x \sim 600 \mu \mathrm{m})$ & 180530 & -26.2 & 2379 & 506 & 229 \\
\hline & Rectangular $(x \sim 716 \mu \mathrm{m})$ & 205468 & -36.4 & 1007 & 377 & $>300$ \\
\hline \multirow{2}{*}{ PMMA $(\sim 1)$} & Triangular $(x \sim 600 \mu \mathrm{m})$ & 204142 & -117.7 & 5149 & 1095 & 664 \\
\hline & Rectangular $(x \sim 600 \mu \mathrm{m})$ & 205468 & -94.2 & 4094 & 871 & 504 \\
\hline MPS ( 1.25) & Rectangular $(x \sim 685 \mu \mathrm{m})$ & 204142 & -378.3 & 10551 & 3440 & 1009 \\
\hline
\end{tabular}


fairly comparable with particles count from the SEM. Generally, the observed disparities in particle counts (from the two approaches) would be attributed to some drift and inconsistencies in positioning of the dispensing tip, and therefore the droplet, on the sensor and to the formation of multilayers. The former is a consequence of misalignment and may have led to spilling or adsorbing of particles along the edges of the beam (cf. inset in Figure 6b), while the latter introduces uncertainties in particle count estimations. This study therefore intends to pick up from here to resolve the aforementioned challenges, and calibrate the cantilever sensors for better comparative particle measurement and count estimations.

\section{Conclusion}

In this work, the droplet dispensing parameters ( $p$ and $t$ ) were optimized and a small water droplet volume of $V_{d}=53.0 \pm 2.7 \mathrm{pL}$ was realized. Furthermore, a droplet evaporation time of $t_{\mathrm{ev}} \approx 40 \mathrm{~s}$ was determined and found to compare well with the theoretical estimation. Particle adsorption on hydrophilic Si substrates and piezoresistive microcantilevers has also been presented, followed by a determination of their mass and number based on both resonant frequency shift measurements and SEM image analysis for particle counting.

\section{Acknowledgments}

W. O. Nyang'au is grateful to the German Federal Ministry for Economic Cooperation and Development (BMZ) for a doctoral scholarship within the Braunschweig International Graduate School of Metrology (B-IGSM). A. Setiono and $P$. Puranto acknowledge support from the Ministry of Research, Technology and Higher Education of the Republic of Indonesia under no. 343/RISET-Pro/FGS/VII/2016 (World Bank Loan No. 8245-ID). M. Bertke is grateful for the support received from Niedersächsisches Vorab, Germany, through the "Quantum- and Nanometrology (QUANOMET)" initiative within the project NP 2-2. The authors are further grateful to Angelika Schmidt, Juliane Breitfelder, Aileen Michalski, and Karl-Heinz Lachmund for their assistance during preparation of research tools and valuable technical assistances.

\section{References}

[1] T. Lindemann and R. Zengerle, "Droplet Dispensing," in Encyclopedia of Microfluidics and Nanofluidics, D. Li, Ed., New York, NY: Springer New York, 2015, pp. 641-652.

[2] T.-J. Yoon et al., "Multifunctional nanoparticles possessing a "magnetic motor effect" for drug or gene delivery," (eng), Angewandte Chemie (International ed. in English), vol. 44, no. 7, pp. 10681071, 2005.

[3] W.-L. Chou, P.-Y. Lee, C.-L. Yang, W.-Y. Huang, and Y.-S. Lin, "Recent Advances in Applications of Droplet Microfluidics," Micromachines, vol. 6, no. 9, pp. 12491271, 2015.

[4] T. P. Burg et al., "Weighing of biomolecules, single cells and single nanoparticles in fluid," (eng), Nature, vol. 446, no. 7139, pp. 1066-1069, 2007.

[5] M. Park, H. Choi, S. J. Kim, C. S. Kim, and T. Kouh, "Resonance Frequency Shift of Microcantilever via Surface Adsorption of Magnetic Nanoparticles," JKMS, vol. 28, no. 2, pp. 49-52, 2018.

[6] W. O. Nyang'au et al., "Adsorption and detection of microparticles using silicon microcantilevers," (to be published in the Journal of Physics: Conference Series, 29th Micromechanics and Microsystems Europe workshop).

[7] C. Ruffert, "Magnetic Bead-Magic Bullet," (eng), Micromachines, vol. 7, no. 2, 2016.

[8] H. S. Wasisto, S. Merzsch, E. Uhde, A. Waag, and E. Peiner, "Handheld personal airborne nanoparticle detector based on microelectromechanical silicon resonant cantilever," Microelectronic Engineering, vol. 145, pp. 96-103, 2015.

[9] M. Bertke et al., "Analysis of asymmetric resonance response of thermally excited silicon micro-cantilevers for masssensitive nanoparticle detection," J. Micromech. Microeng., vol. 27, no. 6, p. 64001, 2017.

[10] A. F. Stalder et al., "Low-bond axisymmetric drop shape analysis for surface tension and contact angle measurements of sessile drops," Colloids and Surfaces A: Physicochemical and Engineering Aspects, vol. 364, no. 1-3, pp. 72-81, 2010.

[11] S. Schmid, L. G. Villanueva, and M. L. Roukes, Fundamentals of 
Nanomechanical Resonators. Cham:

Springer International Publishing, 2016.

[12] J. Arcamone, E. Dujardin, G. Rius, F.

Pérez-Murano, and T. Ondarçuhu,

"Evaporation of femtoliter sessile droplets monitored with nanomechanical mass sensors," (eng), The journal of physical chemistry. B, vol. 111, no. 45, pp. 1302013027, 2007.

[13] H. Y. Erbil, G. McHale, and M. I. Newton, "Drop Evaporation on Solid Surfaces: Constant Contact Angle Mode," Langmuir, vol. 18, no. 7, pp. 2636-2641, 2002.

[14] E. L. Cussler, Diffusion: Mass transfer in fluid systems, 3rd ed. Cambridge, New York: Cambridge University Press, 2009.

[15] C. T. Rueden et al., "ImageJ2: ImageJ for the next generation of scientific image data," (eng), BMC bioinformatics, vol. 18, no. 1, p. 529, 2017. 